\title{
Uterine Microbiota of Dairy Cows With Clinical and Subclinical Endometritis
}

\author{
Meng-Ling Wang, Ming-Chao Liu, Jin Xu, Li-Gang An, Jiu-Feng Wang and \\ Yao-Hong Zhu* \\ Department of Veterinary Clinical Sciences, College of Veterinary Medicine, China Agricultural University, Beijing, China
}

OPEN ACCESS

Edited by:

Suhelen Egan,

University of New South Wales,

Australia

Reviewed by:

Vinicius Machado,

Texas Tech University, United States

Fabio S. Lima,

University of Illinois

at Urbana-Champaign, United States

*Correspondence:

Yao-Hong Zhu

zhu_yaohong@hotmail.com

Specialty section:

This article was submitted to

Microbial Symbioses,

a section of the journal

Frontiers in Microbiology

Received: 29 May 2018

Accepted: 22 October 2018

Published: 06 November 2018

Citation:

Wang M-L, Liu M-C, Xu J, An L-G, Wang J-F and Zhu Y-H (2018) Uterine Microbiota of Dairy Cows With Clinical and Subclinical

Endometritis.

Front. Microbiol. 9:2691 doi: 10.3389/fmicb.2018.02691
The objective of this study was to characterize the uterine microbiota of dairy cows with clinical and subclinical endometritis and to identify the potential bacterial genera as well as their interactions associated with uterine disease. Uterine flush samples $(n=27)$ were collected from 13 healthy, 5 subclinical endometritic (SE), and 9 clinical endometritic (CE) cows at 30 days postpartum. Microbial DNA from uterine flush samples was subjected to sequencing of the 16S rRNA gene on the Illumina MiSeq platform. The uterine microbiota of healthy, SE, and CE cows had similarly complex microbial diversity, and shared 293 of 445 operational taxonomic units. However, endometritic and healthy cows could be discriminated by the relative abundance of bacterial genera. In CE cows, the uterine microbiota was characterized by increased abundance of Fusobacterium and unique presence of Trueperella and Peptoniphilus. For SE cows, known intrauterine pathogens were almost absent and the uterine microbiota was characterized by enrichment of Lactobacillus and Acinetobacter. Analysis of correlations between bacterial genera showed that the uterine microbiota exhibited two co-occurrence groups (i.e., the Lactococcus and the Fusobacterium COGs), indicating that the synergistic effect by co-occurred bacteria may be an important aspect of pathogenesis. Our findings support that common uterine pathogens are not associated with subclinical endometritis at 30 days postpartum and indicate the need of investigating the role of commensal bacteria such as Lactobacillus, and Acinetobacter in the inflammatory process of uterine endometrium.

Keywords: uterus, microbiota, uterine flush, cow, endometritis

\section{INTRODUCTION}

Endometritis is one of the most important causes of infertility in dairy cows, resulting in high economic losses in the dairy industry (Sheldon et al., 2009; Wagener et al., 2017). Endometritis is a superficial inflammation of the endometrium without systemic signs (Sheldon et al., 2006). Clinical endometritis is defined as the presence of purulent or mucopurulent vaginal discharge at 21 or more days postpartum, accompanied by a prominent leukocyte infiltration into the uterine lumen. Subclinical endometritis is characterized by an increased proportion of polymorphonuclear neutrophils (PMN) cells in the endometrium, with the absence of signs of clinical endometritis (Kasimanickam et al., 2004). Indeed, a broad diversity of bacteria, including potential pathogens, can be observed in the uterus of $80-100 \%$ of dairy cows during the first 2 weeks postpartum 
(Sheldon et al., 2009). Depending on the balance between the immune response and uterine infection, about $25-40 \%$ of cows develop metritis within first 3 weeks postpartum (Markusfeld, 1987; Drillich et al., 2001); subsequently, 15-20\% of cows develop clinical endometritis, and 30\% develop subclinical endometritis beyond 3 weeks postpartum (LeBlanc et al., 2002; Gilbert et al., 2005; Cheong et al., 2011). Postpartum endometritis has a negative effect on reproductive performance as it delayed resumption of ovarian cycles, prolonged postpartum luteal phases, increased days to first service and days open, and decreased the conception rate (Kasimanickam et al., 2004; Ribeiro et al., 2013).

Studies using culture-dependent methods have identified several uterine pathogens associated with endometritis, including Escherichia coli, Trueperella pyogenes, Fusobacterium necrophorum, and Prevotella species (Dohmen et al., 1995; Williams et al., 2005; Carneiro et al., 2016). Members of the genera Bacillus, Streptococcus, and Enterococcus, in addition to coagulase-negative Staphylococci, are among the most frequently isolated intrauterine bacteria and have been described as potential or opportunistic pathogens (Wagener et al., 2014, 2015; Carneiro et al., 2016). Recently developed cultureindependent molecular approaches based on sequencing have expanded our current knowledge of the uterine microbiome in cows with metritis, pyometra, and endometritis (Santos and Bicalho, 2012; Jeon et al., 2015; Knudsen et al., 2015, 2016; Bicalho et al., 2017a,b). T. pyogenes was the most important bacteriological risk factor for clinical endometritis, but not for subclinical endometritis (Prunner et al., 2014b). Bacterial growth density on the agar plates increased the risk for subclinical endometritis (Prunner et al., 2014a). Knowledge regarding the uterine microbiota in subclinical endometritic (SE) cows, mainly gleans from studies based on routine microbial isolation and culture techniques. The results from culture-based studies indicated that uterine infections with known pathogens play a minor role in SE cows (Sens and Heuwieser, 2013; Madoz et al., 2014; Prunner et al., 2014a,b).

In this study, we explored the uterine microbiota from the uterine flush samples of healthy, SE and clinical endometritic (CE) cows in an attempt to identify bacterial genera that were associated with endometritis via 16S rRNA gene profiling by high-throughput sequencing. Furthermore, we performed the cooccurrence network analysis to identity potential interactions between genera in the uterine microbiota of dairy cows. The data generated through this work might ultimately facilitate the development of efficient disease prevention and intervention strategies.

\section{MATERIALS AND METHODS}

\section{Experimental Design and Sampling}

All animal procedures were performed in accordance with the approved guidelines and regulations, and the ethical approval of the Animal Ethics Committee of the China Agricultural University (CAU20140728-2).
The study was conducted on a commercial dairy farm in Beijing, China. The herd consisted of 800 milking Holstein dairy cows with an average milk production of 9,527 $\mathrm{kg}$ per lactation. A total of 38 cows were enrolled in the study. During the sample collection period, nine cows were excluded because of a systemic antibiotic treatment. Reasons for these antibiotic treatments were mastitis $(n=5)$, metritis $(n=2)$, pyometra $(n=1)$, vaginal lacerations $(n=1)$. Another two cows were excluded because of the poor quality of cytological smears. Therefore, the complete data set of 27 cows was used for statistical analyses. The average parity was 3.3, and an average body condition score (BCS) was 3.1 (see Supplementary Table S1). No differences were found among groups in parity and BCS. On day 30 postpartum, all cows underwent a vaginal inspection, rectal palpation of the uterus, endometrial cytological examination, and their overall condition was recorded. The BCS was evaluated on a scale from 1 to 5 . The cows were selected for the study dependent on the health status of the uterus. Vaginal discharge was scored as previously described (Williams et al., 2005): score 0 with clear or translucent mucus; score 1 with mucus containing flecks of white or off-white pus; score 2 with less than $50 \%$ white or off-white mucopurulent material in the mucus; and score 3 with more than $50 \%$ purulent material, usually white or yellow, but occasionally sanguineous in the mucus. Cows exhibiting mucopurulent or worse (purulent or foul) vaginal discharge without signs of systemic illness as well as the presence of purulent material within the uterine lumen were classified as having clinical endometritis $(n=9)$. In the absence of purulent vaginal discharge, cows with the proportion of $\mathrm{PMN} \geq 18 \%$ by cytological examination were classified as having subclinical endometritis $(n=5)$. Cows with a clear or translucent vaginal discharge that was not fetid or mucopurulent and with the proportion of PMN $<18 \%$ by cytological examination were classified as healthy $(n=13)$.

Uterine flush samples were collected from cows at 30 days postpartum. Uterus was flushed with saline, using a pipette (Santos and Bicalho, 2012). Briefly, each cow was restrained and the perineum area was disinfected with $70 \%$ ethanol. The infusion pipette covered with a protective plastic sheath was introduced into the cervix; the sheath was subsequently ruptured and the clean pipette tip was manipulated through the cervix into the uterus. The pipette has a deflated balloon in the tip. Once inside the uterus, the balloon was inflated to prevent vaginal or cervix contamination. A total of $30 \mathrm{~mL}$ of sterile saline was infused into the uterus, agitated gently, and a sample of the fluid aspirated. Recovered fluid was transferred to two polypropylene centrifuge tubes and placed on ice for transport to the laboratory within $4 \mathrm{~h}$. One tube of each uterine flush sample was stored at $-80^{\circ} \mathrm{C}$ for DNA extraction, the other tube was taken for cytological examination. Briefly, the uterine flush samples were centrifuged at $750 \times g$ for $10 \mathrm{~min}$. After discarding the supernatant, the remaining pellets were re-suspended and smeared onto microscope slides. The slides were fixed and stained with Diff Quick. A total amount of 300 cells (endometrial epithelial cells and PMNs) were counted under a microscope by $\times 400$ magnification to determine the proportion of PMN. A proportion of $18 \%$ PMN was set as the threshold for the 
diagnosis of subclinical endometritis in cows with clear vaginal discharge (Kasimanickam et al., 2004).

\section{DNA Extraction and Sequencing}

Uterine flush samples collected from 27 cows were prepared for DNA extraction and sequencing of the 16S rRNA gene. An aliquot of $2 \mathrm{~mL}$ was centrifuged for $30 \mathrm{~min}$ at 15,000 $\times g$ at $4^{\circ} \mathrm{C}$. The supernatant was discarded, the pellets were suspended in $200 \mu \mathrm{L}$ of phosphate-buffered saline (PBS) to concentrate microbial cells. The PBS suspension was used to isolate bacterial genomic DNA using a QIAamp DNA minikit (Qiagen, Valencia, CA, United States) according to the manufacturer's protocol, with a minor modification: before AL buffer was added, samples were incubated with $400 \mathrm{mg}$ of lysozyme for $12 \mathrm{~h}$ at $56^{\circ} \mathrm{C}$ to maximize bacterial DNA extraction. The purity and concentration of genomic DNA were determined using a spectrophotometer (Nanodrop 1000; Thermo Scientific, Waltham, MA, United States). Genomic DNA was amplified by PCR with primers that target the V3 and V4 hypervariable regions of the $16 \mathrm{~S}$ rRNA gene. The forward primer sequence was $338 \mathrm{~F}\left(5^{\prime}-\right.$ ACTCCTACGGGAGGCAGCAG-3'), and the reverse primer sequence was 806R ( $5^{\prime}$-GGACTACHVGGGTWTCTAAT- $\left.3^{\prime}\right)$. An eight-base sequence unique to each sample preceded the primers for sample identification using a HotStarTaq Plus master mix kit (Qiagen) according to a custom Illumina preparation protocol. Amplicons were excised from 1.5\% agarose gels and purified using the AxyPrep DNA Gel Extraction Kit (Axygen Biosciences, Union City, CA, United States) according to the manufacturer's protocol and quantified using ST fluorometer (Promega, Madison, WI, United States). A composite sample library for sequencing was created by combining equimolar ratios of amplicons from the individual samples. The composite sample library was cleaned using an UltraClean-htp 96-well PCR cleanup kit (Mo Bio Laboratories, Carlsbad, CA, United States). Pooled amplicons were paired-end sequenced (PE $2 \times 250)$ on an Illumina MiSeq platform according to standard protocols.

\section{Sequence Analysis}

The sequence data were deposited in the NCBI Sequence Read Archive database (accession number SRP102408). Raw fastq files were demultiplexed and quality-filtered using the Quantitative Insights Into Microbial Ecology (QIIME) (Caporaso et al., 2010) with the following criteria: (i) The 250 bp reads were truncated at any site receiving an average quality score $<20$ over a 50 bp sliding window, discarding the truncated reads that were shorter than $50 \mathrm{bp}$; (ii) there were exact barcode matching and a maximum of two nucleotide mismatches to primer sequences; (iii) no ambiguous bases; and (iv) only sequences that overlap longer than $10 \mathrm{bp}$ were assembled according to their overlap sequence. Reads that could not be assembled were discarded. Chimeras were checked and excluded using the Uchime algorithm (version 4.2.40 ${ }^{1}$ ). The resulting high-quality sequences were clustered into operational taxonomic units (OTUs) at 97\% identity level using Usearch 6.1 methodology

${ }^{1}$ http://drive5.com/usearch/manual/uchime_algo.html (version 6.1.544). All singleton OTUs were removed in an attempt to discard the majority of chimera sequences. The OTUs that reached at a $97 \%$ similarity level were used for alpha diversity, Good's coverage, Venn diagram, rarefaction, and rank abundance curve analysis using Mothur (version 1.31.2). Sequences were subsampled to the lowest number of sequences found in all samples (9,666 reads) to evaluate alpha diversity. Alpha diversity was assessed by Shannon index, and the number of observed OTUs. The principal coordinate analysis (PCoA) based on BrayCurtis distance was performed using OTUs from each sample and plotted by the vegan package in $\mathrm{R}$.

Taxonomic classification of the representative sequence for each OTU was performed using the Ribosomal Database Project classifier ${ }^{2}$ (Release 11.1) with a cutoff of $80 \%$ homology against the Silva Gold reference database ${ }^{3}$ (Release 128). OTUs were grouped at different levels of classification (phylum, class, order, family, and genus), at each level, unclassified OTUs were grouped together by the highest available resolution. A heat map was generated with average linkage hierarchical clustering of BrayCurtis distance based on the relative abundances of genera per animal.

The correlations between the 28 most abundant genera were calculated using pairwise Spearman's rank based on relative abundance in the $\mathrm{R}$ stats package. The correlation matrix was visualized and clustered in $\mathrm{R}$ using the Made4 package and Heatplot function, and hierarchical Ward-linkage clustering was used to define genus co-occurrence groups (COGs) (Biagi et al., 2016). The correlations were visualized in network interface with Cytoscape software (Shannon et al., 2003). The nodes represented genera, and the size of each node is proportional to the average relative abundance. The edges between nodes represented significant $(P<0.05)$ correlations, and the thickness of edge is proportional to the correlation strength.

\section{Statistical Analysis}

Statistical analysis was carried out using R Statistical Language and GraphPad Prism (version 7.0) software. All continuous variables, such as relative abundance of bacteria, Shannon index, and the number of observed OTUs were analyzed using the Kruskal-Wallis analysis of variance on ranks, followed by Dunn's test to adjust for multiple comparisons. Permutational multivariate analysis of variance (PERMANOVA) was performed using the vegan package in $\mathrm{R}$. Correlations were calculated by Spearman's rank correlation in the $\mathrm{R}$ stats package. The linear discriminant analysis (LDA) effect size (LEfSe) ${ }^{4}$ method was used to identify indicator bacteria differentiating the uterine microbiota between healthy and endometritic cows, which emphasizes both statistical significance and biological relevance. LEfSe uses the Kruskal-Wallis rank sum test with a normalized relative abundance matrix to detect differentially abundant features between groups and performs LDA to estimate the effect size of each feature (Segata et al., 2011). A significance level (alpha) of 0.05 and an effect size threshold of 3 were used for all

\footnotetext{
${ }^{2}$ http://rdp.cme.msu.edu/

${ }^{3} \mathrm{http}: / /$ www.arb-silva.de

${ }^{4}$ http://huttenhower.sph.harvard.edu/lefse/
} 
indicators discussed. All tests for significance were two-sided, and a significance level of 0.05 was considered statistically significant in this study.

\section{RESULTS}

\section{Bacterial Diversity of the Uterine Microbiota}

To profile the bovine uterine microbiota, we performed $16 \mathrm{~S}$ rRNA sequencing for 27 uterine flush samples using an Illumina MiSeq platform. A total of 468,846 raw sequence reads were obtained. Following quality trimming and chimera checking, 392,246 high quality reads remained, accounting for $83.7 \%$ of the valid reads. The rarefaction curves (Supplementary Figure S1 and Figure 1A) showed that this sequencing depth was sufficient to cover the overall bacterial diversity. The value of Good's coverage of each cow were greater than $99 \%$, indicating that this sequencing method can characterize the true composition of uterine microbiota. The rank-abundance curves showed that a few species accounted for more than $1 \%$ abundance in the uterine microbiota (Figure 1B). Species diversity was measured as Shannon index, species richness was calculated as the number of OTUs. No differences were found among groups in species diversity and species richness (Figures 1C,D). Venn diagram showed that 293 of the 445 total OTUs were shared among groups (Figure 1E). These 293 shared OTUs dominated the uterine microbiota, represented $99.68 \%, 99.39 \%$, and $98.67 \%$ of the total OTUs abundance in the healthy, SE, and CE cows, respectively. The uterine microbiota of healthy, SE, and $\mathrm{CE}$ cows had similar level of microbial diversity and shared most bacterial species (Figures 1C-E). The PCoA analysis showed that the samples from $\mathrm{CE}$ cows could be separated from healthy cows, although two CE samples were clustered with the healthy group (Figure 1F). The PERMANOVA analysis of the uterine samples showed significant differences in community composition between the healthy and CE groups $(P=0.004$, $\left.R^{2}=0.182\right)$, and no significant difference in community composition between the healthy and SE groups $(P=0.167$, $\left.R^{2}=0.097\right)$.

\section{Endometritis-Associated Alterations in the Uterine Microbiota}

A total of 17 phyla were identified in the uterine microbiota of all samples. Taxonomic assignment showed that the Firmicutes (76.7\%), Proteobacteria (8.1\%), Actinobacteria (5.9\%), Bacteroidetes (4.6\%), Fusobacteria (4.3\%), and Tenericutes $(0.2 \%)$ were the six most abundant phyla in the uterus of all dairy cows, accounting for $99.8 \%$ of the total abundance. In total, 206 genera were identified across all the samples. A heat map of the 40 most abundant genera (at $0.1 \%$ or greater abundance within either group) is shown in Figure 2, accounting for $99.66 \%, 99.48 \%$, and $98.61 \%$ of the total genera abundance in the healthy, SE, and CE groups, respectively. Lactococcus, Bacillus, Solibacillus, Pseudomonas, and Arthrobacter were the five most abundant genera with little variations in the uterus of healthy and
SE cows (Supplementary Table S4). In CE cows, the Lactococcus, Bacillus, Fusobacterium, ratAN060301.norank, and Solibacillus were the top five abundant genera. The genus-level heat map analysis showed an obvious shift in the uterine microbiota of $\mathrm{CE}$ cows, with an increase in the genera Fusobacterium, Parvimonas, Porphyromonas, Peptoniphilus, Helcococcus, Trueperella, and ratAN060301.norank (OTU154, uncultured Porphyromonas species in the family ratAN060301, order Bacteroidales), compared with healthy and SE cows.

To identify genera associated with endometritis, LEfSe was performed using the 28 most abundant bacterial genera in the uterine microbiota of healthy and endometritic cows. Compared with healthy cows, Fusobacterium, Trueperella, and Peptoniphilus were discriminately enriched (LDA sores > 3.5) in CE cows (Figure 3A and Supplementary Table S2). In SE cows, Lactobacillus, and Acinetobacter were discriminately increased (LDA scores $>3$ ), compared with healthy cows (Figure 3B and Supplementary Table S3).

To further explore the relationship between specific bacteria and clinical endometritis, we stratified cows by vaginal discharge score as a clinical sign and performed a Spearman's rank correlation tests on the relative abundances of the 28 most abundant bacterial genera. The relative abundance of Fusobacterium increased with increasing vaginal discharge score (Spearman's $r_{\mathrm{s}}=0.51, P=0.006$ ) (Figure 4A). Likewise, the relative abundance of Trueperella increased with increasing vaginal discharge score (Spearman's $r_{\mathrm{s}}=0.48 ; P=0.017$ ) (Figure 4B).

We also compared the relative abundances of the 28 most abundant genera in the uterine microbiota using Kruskal-Wallis rank-sum test (Figure 4 and Supplementary Table S3). Compared with healthy cows, the relative abundance of Fusobacterium increased in CE cows, the abundance of Lactococcus, Bacillus, Solibacillus, Pseudomonas, and Arthrobacter decreased $(P<0.05)$. Lactobacillus and Acinetobacter were more abundant in SE cows than in healthy cows $(P<0.05)$. The abundance of Fusobacterium and Trueperella decreased in SE cows, compared with those in $\mathrm{CE}$ cows $(P<0.05)$. No differences in the abundance of Lactococcus, Bacillus, Solibacillus, Pseudomonas, and Arthrobacter were observed between the healthy and SE cows.

\section{Interactions Between Bacterial Genera in the Uterine Microbiota}

We evaluated the correlations between the 28 most abundant bacterial genera, and performed hierarchical Ward-linkage clustering of the correlations to define co-occurrence group (COG) (Figure 5A). The distribution of COGs differed significantly among groups, determined by PERMANOVA analysis using the Bray-Curtis dissimilarity (Fusobacterium COG $F=2.31, P=0.003$; Lactococcus COG $F=2.39$, $P=0.002)$. The Lactococcus COG represented the majority of the uterine microbiota in terms of high predominate abundance, accounted for $94.1 \%, 91.1 \%$, and $66.1 \%$ in the healthy, SE, and CE groups, respectively. The Fusobacterium COG (accounted for 28.39\%) was exclusively enriched in the 

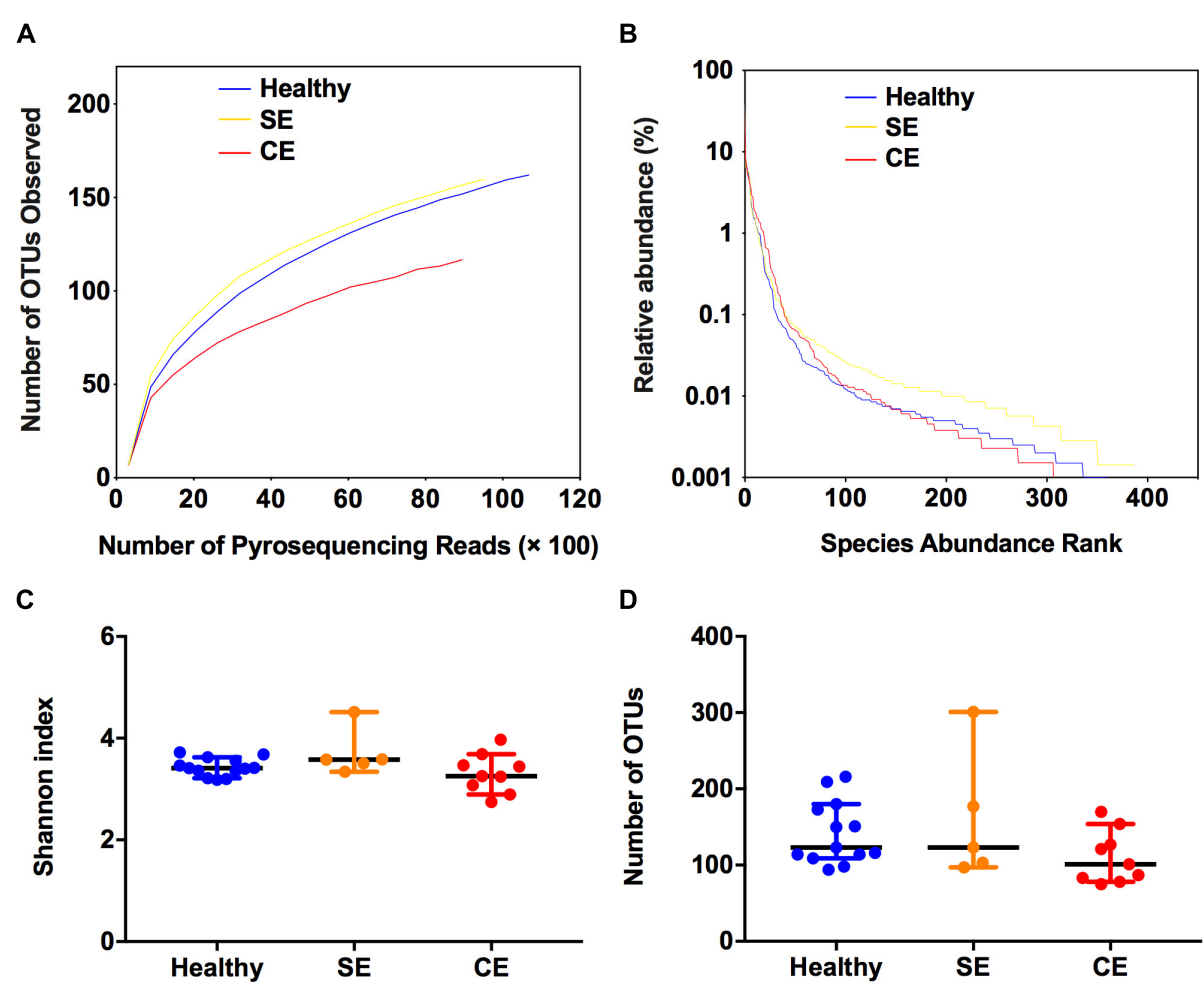

D
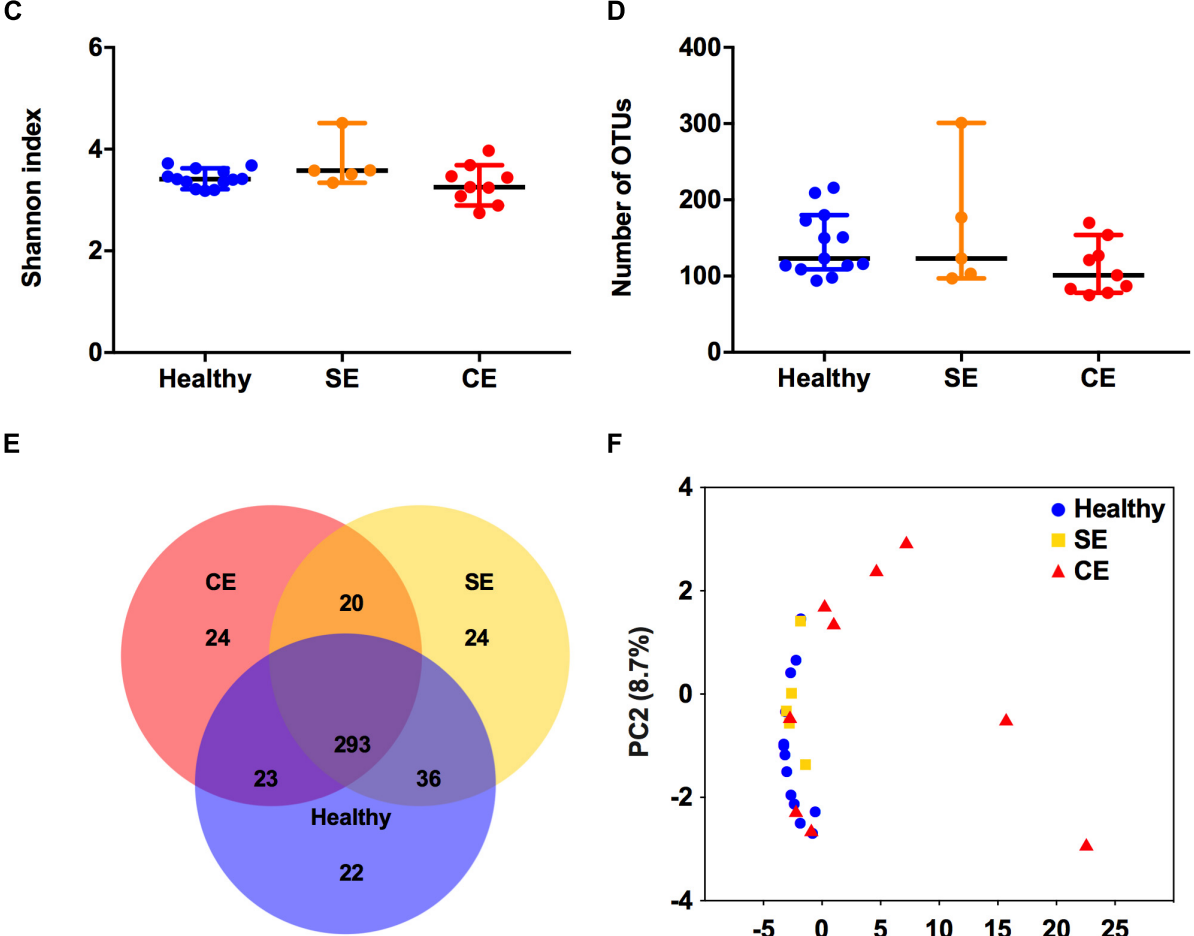

$\mathbf{F}$

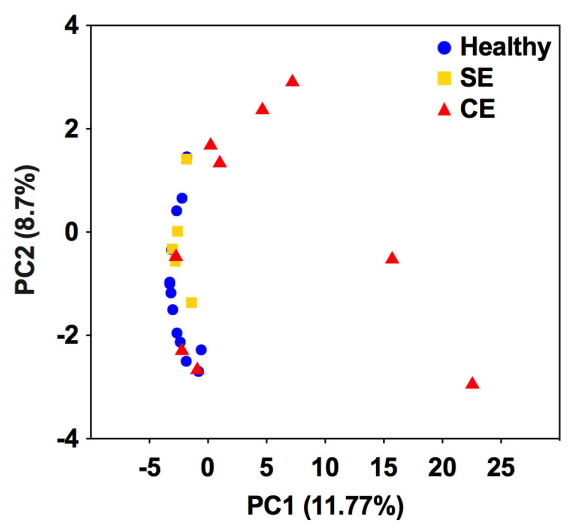

FIGURE 1 | Structural comparison of the uterine microbiota. The rarefaction curves (A), rank abundance curves (B), Shannon index (C), and the number of operational taxonomic units (OTUs) (D) were used to estimate alpha diversity of the uterine microbiota in healthy, SE, and CE cows. Symbols represent data from individual cows, data shown as median with $95 \% \mathrm{Cl}$. (E) Venn diagram illustrating the common and exclusive OTUs in the uterine microbiota of the three groups. (F) Principal coordinate analysis based on the relative abundance of OTUs with Bray-Curtis distances, showing the differences between each individual cow. Healthy, healthy cows, $n=13$; SE, subclinical endometritic cows, $n=5$; CE, clinical endometritic cows, $n=9$.

uterine microbiota of CE cows (Figure 5B). Fusobacterium, Porphyromonas, Trueperella, Helcococcus, and Peptoniphilus represented $10.58 \%, 3.05 \%, 2.24 \%, 1.55 \%$, and $0.66 \%$ of the total bacterial population in CE cows, while little abundance was found in the uterine microbiota of healthy and SE cows (representing less than $0.2 \%$ of the total bacterial population) (Supplementary Table S4). At species level, sequences from F. necrophorum (OTU430), T. pyogenes (OTU99), Helcococcus ovis (OTU311) and Peptoniphilus indolicus (OTU295) were identified in this study (Supplementary Table S5).
A number of positive correlations were found among genera from the same COG (Figure 5C and Supplementary Table S6). Major uterine pathogens such as Fusobacterium, Trueperella, Bacteroides, and Porphyromonas along with other uterine pathogens such as Peptoniphilus and Helcococcus belonged to the Fusobacterium COG, and had positive correlations. Fusobacterium showed a positive correlation with Trueperella (Spearman's $r_{\mathrm{s}}=0.43, P=0.026$ ), which was positively correlated $(P<0.05)$ with Peptoniphilus, Helcococcus, ratN060301C.norank, and Bacteroides. Helcococcus 


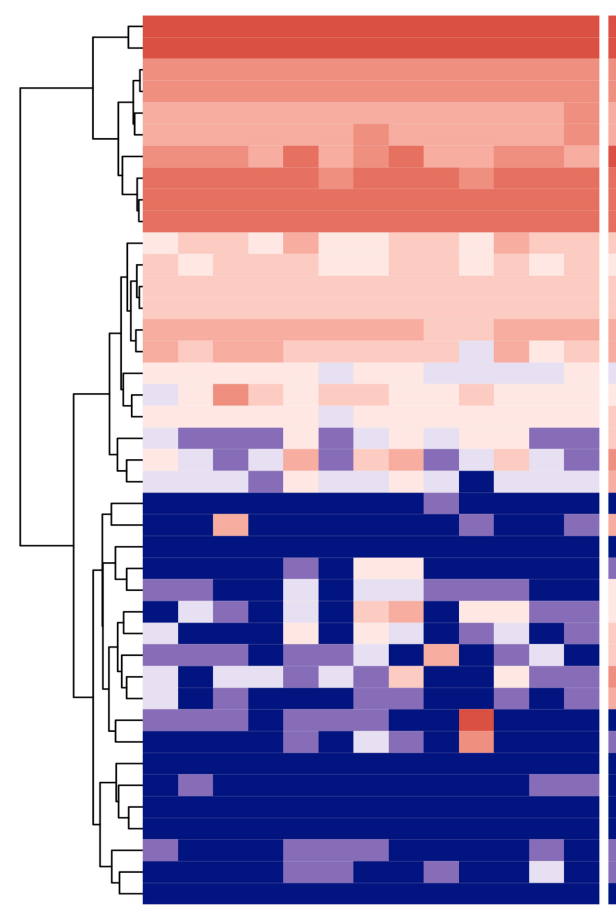

Healthy

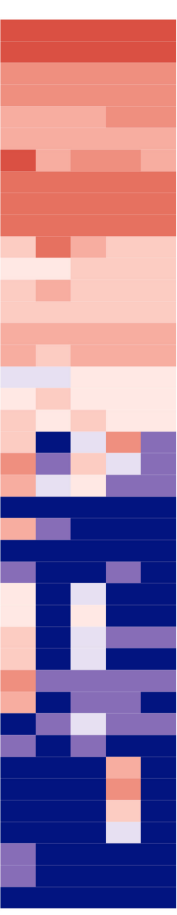

SE

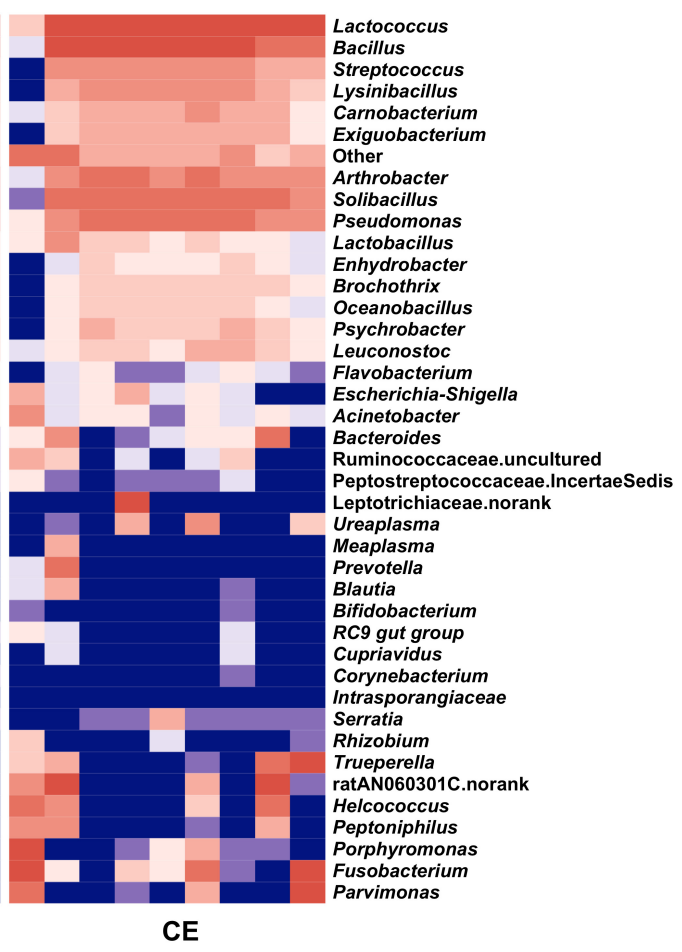

CE

Relative abundance (\%)

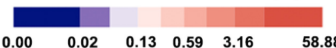

FIGURE 2 | Heat map with average linkage clustering based on Bray-Curtis distance showing the relative abundances of the top 40 genera in each cow. The relative abundance of each genus is indicated by a gradient of color from blue (low abundance) to red (high abundance). Healthy, healthy cows, $n=13$; SE, subclinical endometritic cows, $n=5$; CE, clinical endometritic cows, $n=9$.

and ratN060301C.norank were positively correlated $(P<0.05)$ with Peptoniphilus. Helcococcus was also positively correlated $(P<0.05)$ with ratN060301C.norank and Porphyromonas. Bacteroides was also positively correlated $(P=0.002)$ with Ruminococcaceae uncultured. Fusobacterium showed a positive correlation with Parvimonas $(P=0.003)$. Within the Lactococcus COG, Arthrobacter was positively correlated with Lactobacillus (Spearman's $r_{s}=0.56, P=0.018$ ), and with Psychrobacter (Spearman's $\left.r_{s}=0.80, P=0.00004\right)$. Arthrobacter, Psychrobacter, Bacillus, Solibacillus, and Pseudomonas were positively correlated with many bacteria including Psychrobacter, Carnobacterium, Exiguobacterium, and Brochothrix.

Negative correlations were found between genera from the Lactococcus COG and the Fusobacterium COG (Figure 5C and Supplementary Table S6). The abundance of Fusobacterium was negatively correlated with the abundance of Arthrobacter (Spearman's $r_{s}=-0.53, P=0.028$ ), and with the abundance with Psychrobacter (Spearman's $r_{s}=-0.64, P=0.0003$ ). Psychrobacter, Bacillus, Solibacillus, and Pseudomonas were negatively $(P<0.05)$ correlated with Trueperella. Genera with negative correlations with Fusobacterium and/or Trueperella, comprising Arthrobacter, Psychrobacter, Bacillus, Solibacillus, and Pseudomonas, were positively correlated $(P<0.05)$ with many other bacteria including Psychrobacter, Carnobacterium, Exiguobacterium, and Brochothrix.

\section{DISCUSSION}

In the present study, we investigated the disease-related alterations as well as bacteria interactions in the uterine microbiota of CE and SE cows. Previous studies have described the diversity and complexity of the bacterial community in the postpartum uterus of dairy cows (Santos and Bicalho, 2012; Jeon et al., 2015; Knudsen et al., 2015, 2016; Bicalho et al., 2017a,b). Here, we performed co-occurrence analysis to obtain a comprehensive understanding of the complex bacterial interactions in bovine uterus.

We demonstrated that Fusobacterium, Trueperella, and Peptoniphilus were associated with clinical endometritis, and Fusobacterium and Trueperella were positively correlating with purulent vaginal discharge. Notably, CE-associated genera Fusobacterium, Trueperella, and Peptoniphilus, along with other pathogens such as Porphyromonas, Parvimonas, Bacteroides, and Helcococcus were found to belong to the Fusobacterium COG, and had positive correlations. Therefore, it is likely that Fusobacterium acts synergistically with Trueperella, Porphyromonas, Parvimonas and other bacteria, to cause dysbiosis in the uterine microbiota of CE cows. F. necrophorum, and $T$. pyogenes have been recognized as the major uterine pathogens associated with metritis, endometritis and purulent vaginal discharge (Dohmen et al., 1995; Williams et al., 
A

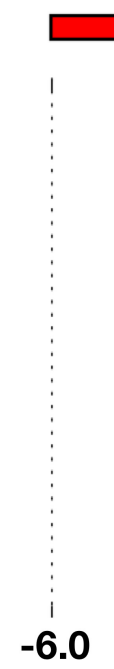

CE

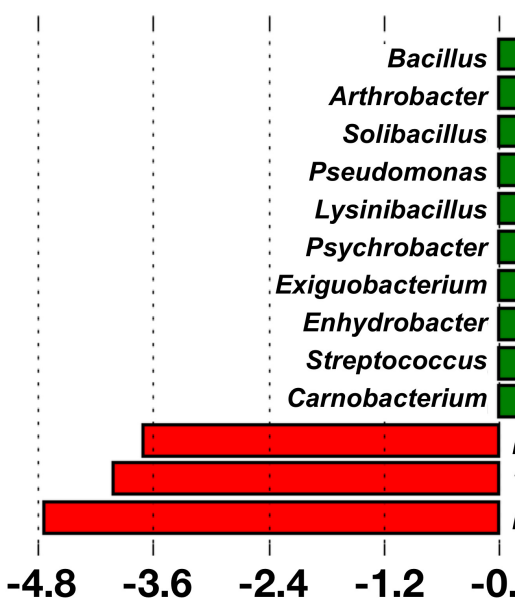

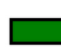

\section{Healthy}

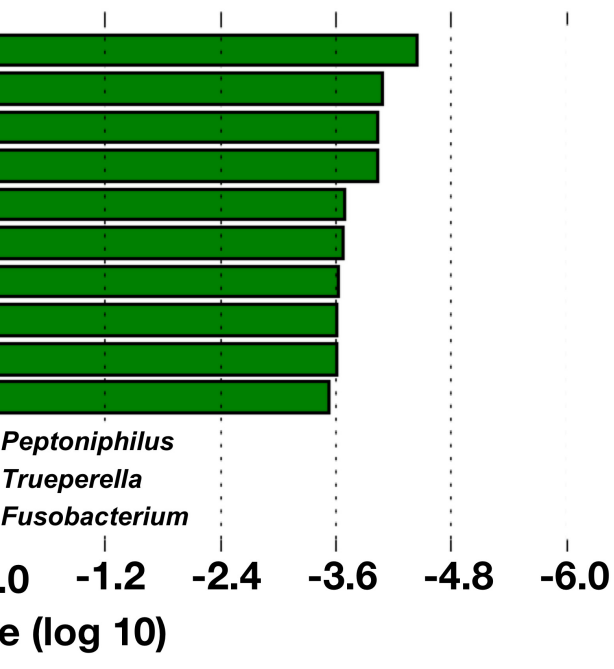

B $\square$ SE

$\square$ Healthy

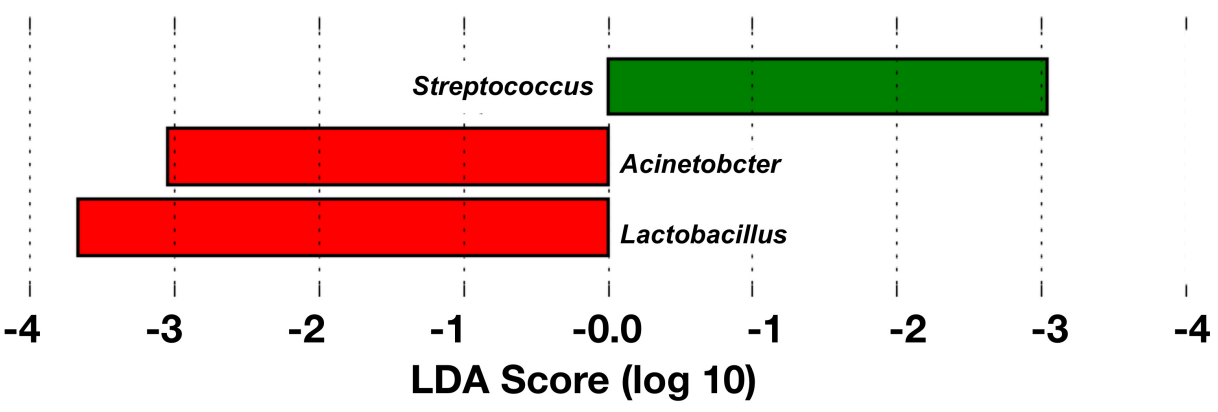

FIGURE 3 | The linear discriminant analysis (LDA) effect size plots showing the differences in the uterine microbiota between healthy cows and CE cows (A), and between healthy cows and SE cows (B). The histogram shows the LDA effect size computed for features at genus level. Healthy-enriched genera are indicated with positive LDA scores (green), and genera enriched in CE or SE cows are indicated with negative LDA scores (red). Only genera meeting a significant level of 0.05 and an effect size threshold of 3 are plotted. Healthy, healthy cows, $n=13$; SE, subclinical endometritic cows, $n=5$; CE, clinical endometritic cows, $n=9$.

2005; Bicalho et al., 2012; Prunner et al., 2014b). It has been proposed that Trueperella and the Gram-negative anaerobes Fusobacterium, Bacteroides, and Porphyromonas act synergistically to cause metritis and endometritis in the uterus (Bonnett et al., 1991; Bicalho et al., 2012; Prunner et al., 2014b). T. pyogenes causes cytolysis in the endometrium by secreting pyolysin (Amos et al., 2014). F. necrophorum produces leukotoxins (Nagaraja et al., 2005), Bacteroides produces short-chain fatty acids (Rotstein, 1993), and Porphyromonas levii produces an immunoglobulin protease that inhibits phagocytosis (Lobb et al., 1999). It is widely believed that $T$. pyogenes support $F$. necrophorum growth and colonization by producing an unknown growth factor (Dadarwal et al., 2017). A synergy between F. necrophorum and Porphyromonas levii has been hypothetically suggested for their co-localization in the lamina propria of the uterus (Karstrup et al., 2017a). It is therefore plausible that uterine pathogens might assist each other in avoiding uterine defense mechanisms and interact to facilitate colonization of the endometrium. Similar cooperative interactions between pathogens were also observed in cows with metritis or purulent vaginal discharged (Bicalho et al., 2012, 2017a,b; Jeon et al., 2015, 2017). Pathogenic bacteria (such as Trueperella spp., Fusobacterium spp.) were also present in the uterus of virgin heifers and of pregnant cows (Karstrup et al., 2017b,c; Moore et al., 2017). Collectively, the co-occurrence of uterine pathogens could be considered of major importance in the development of uterine infection. The cooperative interspecies signaling and mechanism behind synergisms need to be elucidated.

Contrast with clinical endometritis, cows with subclinical endometritis harbored a small proportion of the Fusobacterium COG, constituting only $1.5 \%$ of the total number of sequences. Fusobacterium, Trueperella were rarely detected in samples from SE cows. Early culture-based study demonstrated that subclinical 

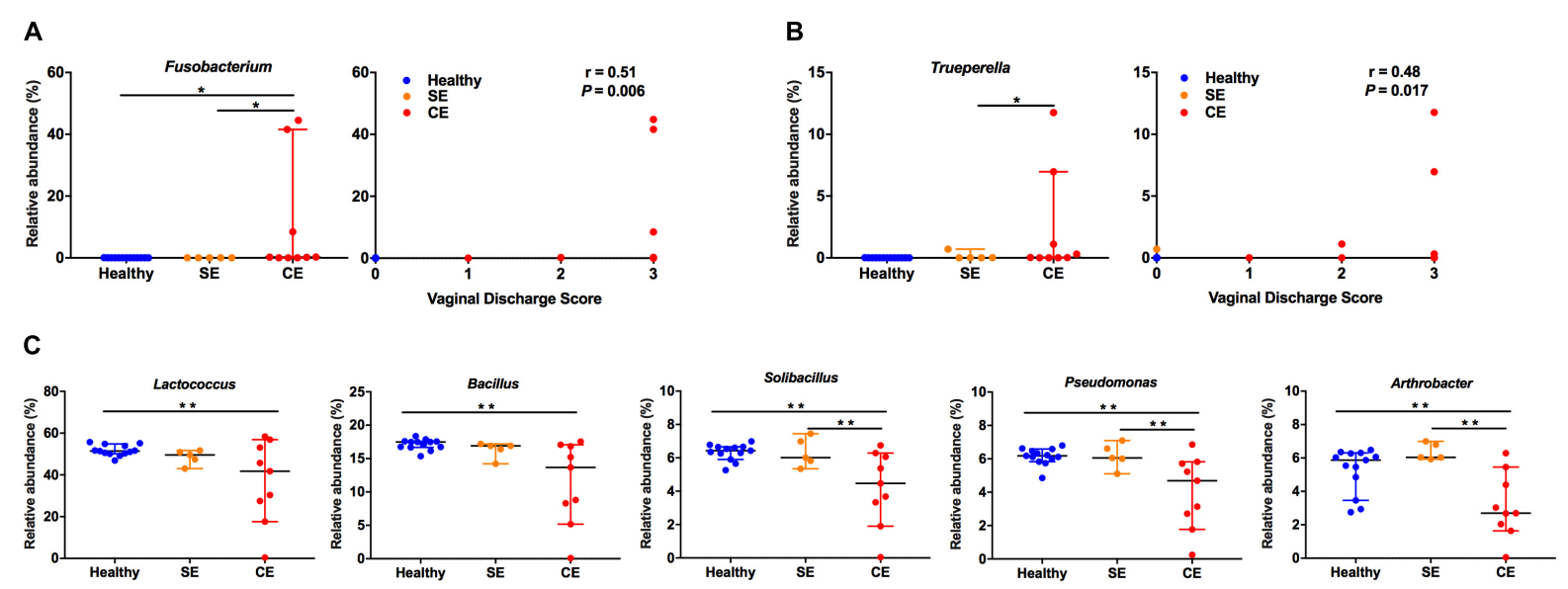

FIGURE 4 | The relative abundance distribution of Fusobacterium (A), Trueperella (B) among groups, and correlations between vaginal discharge scores. Cows were assigned to four groups according to vaginal discharge score: 0, clear or translucent mucus; 1, clear discharge with flecks of pus; 2, mucopurulent, not fetid discharge; 3, purulent or fetid discharge. $r$, Spearman's coefficient. (C) Analysis of the relative abundance of Lactococcus, Bacillus, Solibacillus, Pseudomonas, and Arthrobacter in healthy, SE and CE cows by Kruskal-Wallis rank-sum test. Symbols represent data from individual cows, data shown as median with $95 \%$ Cl. Healthy, healthy cows, $n=13$; SE, subclinical endometritic cows, $n=5$; CE, clinical endometritic cows, $n=9$. ${ }^{*} P<0.05$, ${ }^{* *} P<0.01$.
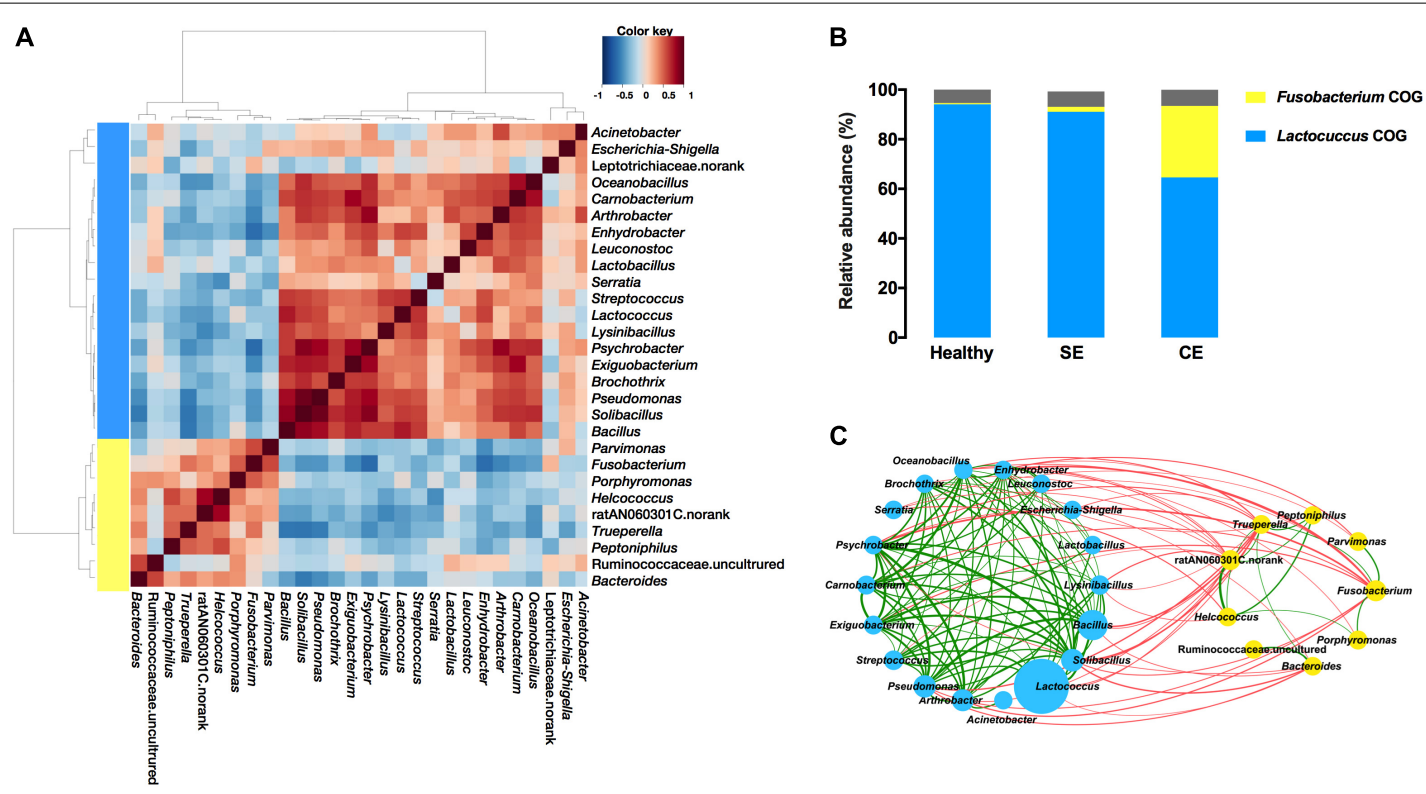

FIGURE 5 | Co-occurrence groups (COGs) assignment depended on heat plot (A) showing Spearman correlations between genera, clustered by Ward-linkage hierarchical clustering. (B) The average cumulative abundance of genera in each COG for healthy, SE and CE cows is also plotted. Colors are indicative of the two identified COGs: Lactococcus COG (blue) and Fusobacterium COG (yellow). The proportion of genera not considered in the COG assignment is colored gray. (C) The network plot showing correlations between bacterial genera in the uterine microbiota of all cows (C). The nodes represent the genera; the circle size of each node is proportional to its average relative abundance in all samples. Blue and yellow nodes indicate genera belong to Lactococcus COG and Fusobacterium COG, respectively. The edges between nodes represent significant $(P<0.05)$ correlations between genera, the thickness of edge is proportional to the correlation strength (see Supplementary Table S5). Green and red edges indicate positive and negative correlations, respectively. Healthy, healthy cows, $n=13$; SE, subclinical endometritic cows, $n=5$; CE, clinical endometritic cows, $n=9$.

endometritis at 21 days postpartum was not associated with T. pyogenes (Prunner et al., 2014a). Early culture-based study also found that $T$. pyogenes were frequently isolated from CE cows, and no bacteria were isolated from SE cows (Madoz et al., 2014). Our observations by sequencing of 16S rRNA gene corroborate previous observations, supporting that uterine infections with major pathogens play a minor role in SE cows compared with $\mathrm{CE}$ cows. The establishment of uterine infections depends on the pathogenicity of invading bacteria and the local immune state. Many factors influence bacteria pathogenicity, including bacterial load, various strains, bacterial virulence factors, and interactions between species etc. (Dadarwal et al., 2017). PMN 
infiltration into the uterine lumen as an indicator of subclinical endometritis is associated with increased endometrial mRNA expression of pro-inflammatory mediators, including cytokines antimicrobial peptides, acute phase proteins and prostaglandins (Fischer et al., 2010; Brodzki et al., 2015). Brodzki et al. (2015) hypothesized that high levels of IL-10 in SE cows contribute to a weakened local immune response in the endometrium, leading to persistent uterine inflammation in the postpartum period (Brodzki et al., 2015). Metabolic imbalances also increase the risk of subclinical endometritis, particularly a negative energy balance, which interferes with an adequate immune response (Wagener et al., 2017). Elevated concentrations of non-esterified fatty acids and beta-hydroxybutyric acid, and a poor BCS increase the risk for subclinical endometritis (Galvão et al., 2010; Heidarpour et al., 2012).

The uterine microbiota of SE cows was characterized by enrichment of Lactobacillus and Acinetobacter. Intravaginal administration of certain strains of Lactobacillus reduced the incidence of uterine diseases in treated cows with enhanced secretory immunoglobulin A production in the vaginal mucus (Deng et al., 2015). Lactobacillus species isolated from the bovine uterus, such as L. amylovorus and L. ruminis, stimulate an immune response without cytotoxic effects (Gärtner et al., 2015). Lactobacillus rhamnosus GR-1 reduces $E$. coli-induced release of pro-inflammatory cytokines in primary bovine endometrial epithelial cells in vitro (Liu et al., 2016). Acinetobacter spp. are observed in the environment (e.g., soil and water) and present in the microbiota of healthy human skin, cattle udder skin, and cattle gut (Yeoman et al., 2018). A. baumannii is the most important species, since it causes serious infections in human (De Amorim and Nascimento, 2017). Acinetobacter strains isolated from samples of milk and milk derivatives could be opportunistic pathogens (Gurung et al., 2013). Acinetobacter strains can be isolated from intrauterine samples collected from repeat breeder cows (Pothmann et al., 2015). Due to the limited information of 16S rRNA sequencing method, it is difficult to define pathogenic strains or harmless commensals associated with subclinical endometritis. In this study, whether these bacterial changes are a cause or a consequence of uterine inflammation is uncertain. Our findings increase the current knowledge of the uterine microbiota in SE cows and provide a basis for future detailed in vitro studies to decipher the affect of these bacteria on immune response in uterine endometrium.

The Fusobacterium COG had negatively correlations with the Lactococcus COG and dramatically more abundant in CE cows, implying that there might be local colonization resistance between the Fusobacterium COG and Lactococcus COG, linked to the microbiota dysbiosis in CE cows. Accordingly, the overgrowth of the Lactococcus COG may result in a decrease of the Fusobacterium COG. Therefore, competition between members of the Fusobacterium COG and Lactococcus COG might restrict the overgrowth of potential pathogens. The composition and function of microbial communities is thought to be largely shaped by interspecies competition for the available resources (Zengler and Zaramela, 2018). The cooperative interactions among co-occurring bacteria, such as metabolite exchanges, could promote group survival under nutritionally challenging conditions (Zelezniak et al., 2015). The different uterine bacterial composition between healthy and diseased cows likely reflect the differing nutritional and physiological conditions. Identifying the source of critical nutrients that support pathogenic overgrowth will be crucial to increasing our understanding of disease pathogenesis and could potentially assist in developing novel treatment strategies (Zengler and Zaramela, 2018). Such competitive and cooperative interactions and mechanisms of establishing infection warrants further detailed studies.

Investigation the uterine microbiota in uterine flush samples from postpartum dairy cows by high throughput sequencing of $16 \mathrm{~S}$ rRNA gene showed that major uterine pathogens such as Fusobacterium, Trueperella, and Peptoniphilus were enriched in the uterine microbiota of $\mathrm{CE}$ cows, but were almost absent in the uterine microbiota of SE cows. Our results demonstrated that known uterine pathogens had co-existence relationships with other bacteria, such as Porphyromonas, Bacteroides, Helcococcus, and Parvimonas, suggesting that their synergistic effects may be crucial contributors in uterine infection. Our findings support that major uterine pathogens are not associated with subclinical endometritis at 30 days postpartum and indicate the need of investigating the role of commensal bacteria such as Lactobacillus, and Acinetobacter in the inflammatory process of uterine endometrium.

\section{AUTHOR CONTRIBUTIONS}

M-LW, M-CL, Y-HZ, and J-FW conceived and designed the experiments. M-LW, M-CL, JX, and L-GA performed the experiments. M-LW performed sequencing analysis and wrote the manuscript.

\section{FUNDING}

The present work was funded by the National Key R\&D Program of China (Project No. 2017YFD0502200), the Program for the Beijing Dairy Industry Innovation Team (BAIC06-2018), and the National Natural Science Foundation of China (Project Nos. 31873034, 31672613, and 31472242).

\section{ACKNOWLEDGMENTS}

We would like to thank Dr. David Robinette for revising and editing the manuscript.

\section{SUPPLEMENTARY MATERIAL}

The Supplementary Material for this article can be found online at: https://www.frontiersin.org/articles/10.3389/fmicb. 2018.02691/full\#supplementary-material 
FIGURE S1 | Rarefaction curves of each individual cow. Healthy, healthy cows, $n=13$; SE, subclinical endometritic cows, $n=5$; and CE, clinical endometritic cows, $n=9$.

TABLE S1 | The basic information of cows included in this study.

TABLE S2 | Key features are statistically different between the uterine microbiota of healthy and clinical endometritic cows.

TABLE S3 | Key features are statistically different between the uterine microbiota of healthy and subclinical endometritic cows.

\section{REFERENCES}

Amos, M. R., Healey, G. D., Goldstone, R. J., Mahan, S. M., Düvel, A., Schuberth, H.-J., et al. (2014). Differential endometrial cell sensitivity to a cholesteroldependent cytolysin links Trueperella pyogenes to uterine disease in cattle1. Biol. Reprod. 90:54. doi: 10.1095/biolreprod.113.115972

Biagi, E., Franceschi, C., Rampelli, S., Severgnini, M., Ostan, R., Turroni, S., et al. (2016). Gut microbiota and extreme longevity. Curr. Biol. 26, 1480-1485. doi: 10.1016/j.cub.2016.04.016

Bicalho, M. L. S., Lima, S., Higgins, C. H., Machado, V. S., Lima, F. S., and Bicalho, R. C. (2017a). Genetic and functional analysis of the bovine uterine microbiota. Part II: purulent vaginal discharge versus healthy cows. J. Dairy Sci. 100, 3863-3874. doi: 10.3168/jds.2016-12061

Bicalho, M. L. S., Machado, V. S., Higgins, C. H., Lima, F. S., and Bicalho, R. C. (2017b). Genetic and functional analysis of the bovine uterine microbiota. Part I: metritis versus healthy cows. J. Dairy Sci. 100, 3850-3862. doi: 10.3168/jds. 2016-12058

Bicalho, M. L. S., Machado, V. S., Oikonomou, G., Gilbert, R. O., and Bicalho, R. C. (2012). Association between virulence factors of Escherichia coli, Fusobacterium necrophorum, and Arcanobacterium pyogenes and uterine diseases of dairy cows. Vet. Microbiol. 157, 125-131. doi: 10.1016/j.vetmic.2011.11.034

Bonnett, B. N., Martin, S. W., Gannon, V. P., Miller, R. B., and Etherington, W. G. (1991). Endometrial biopsy in Holstein-Friesian dairy cows. III. Bacteriological analysis and correlations with histological findings. Can. J. Vet. Res. 55, $168-173$.

Brodzki, P., Kostro, K., Brodzki, A., Wawron, W., Marczuk, J., and Kurek, L (2015). Inflammatory cytokines and acute-phase proteins concentrations in the peripheral blood and uterus of cows that developed endometritis during early postpartum. Theriogenology 84, 11-18. doi: 10.1016/j.theriogenology.2015. 02.006

Caporaso, J. G., Kuczynski, J., Stombaugh, J., Bittinger, K., Bushman, F. D., Costello, E. K., et al. (2010). QIIME allows analysis of high-throughput community sequencing data. Nat. Methods 7, 335-336. doi: 10.1038/nmeth. f.303

Carneiro, L. C., Cronin, J. G., and Sheldon, I. M. (2016). Mechanisms linking bacterial infections of the bovine endometrium to disease and infertility. Phytochem. Lett. 16, 1-7. doi: 10.1016/j.repbio.2015.12.002

Cheong, S. H., Nydam, D. V., Galvão, K. N., Crosier, B. M., and Gilbert, R. O. (2011). Cow-level and herd-level risk factors for subclinical endometritis in lactating Holstein cows. J. Dairy Sci. 94, 762-770. doi: 10.3168/jds.2010-3439

Dadarwal, D., Palmer, C., and Griebel, P. (2017). Mucosal immunity of the postpartum bovine genital tract. Theriogenology 104, 62-71. doi: 10.1016/j. theriogenology.2017.08.010

De Amorim, A. M. B., and Nascimento, J. D. (2017). Acinetobacter: an underrated foodborne pathogen? J. Infect. Dev. Ctries. 11, 111-114. doi: 10.3855/jidc. 8418

Deng, Q., Odhiambo, J. F., Farooq, U., Lam, T., Dunn, S. M., and Ametaj, B. N. (2015). Intravaginal lactic acid bacteria modulated local and systemic immune responses and lowered the incidence of uterine infections in periparturient dairy cows. PLoS One 10:e0124167. doi: 10.1371/journal.pone.0124167

Dohmen, M. J. W., Lohuis, J. A. C. M., Huszenicza, G., Nagy, P., and Gacs, M. (1995). The relationship between bacteriological and clinical findings in cows with subacute/chronic endometritis. Theriogenology 43, 1379-1388. doi: 10 . 1016/0093-691X(95)00123-P
TABLE S4 | Relative abundance of the 28 most abundant genera detected in the uterine of postpartum healthy and endometritic dairy cows at 30 days postpartum.

TABLE S5 | Relative abundance of 28 most abundant species detected in the uterine of postpartum healthy and endometritic dairy cows at 30 days postpartum.

TABLE S6 | Correlations among the 28 most abundant genera detected in the uterus of 27 cows at 30 days post parturition. Only significant $(P<0.05)$ Spearman coefficients of correlation are presented.

Drillich, M., Beetz, O., Pfützner, A., Sabin, M., Sabin, H. J., Kutzer, P., et al. (2001). Evaluation of a systemic antibiotic treatment of toxic puerperal metritis in dairy cows. J. Dairy Sci. 84, 2010-2017. doi: 10.3168/jds.S0022-0302(01)74644-9

Fischer, C., Drillich, M., Odau, S., Heuwieser, W., Einspanier, R., and Gabler, C. (2010). Selected pro-inflammatory factor transcripts in bovine endometrial epithelial cells are regulated during the oestrous cycle and elevated in case of subclinical or clinical endometritis. Reprod. Fertil. Dev. 22, 818-829. doi: 10.1071/RD09120

Galvão, K. N., Flaminio, M. J. B. F., Brittin, S. B., Sper, R., Fraga, M., Caixeta, L., et al. (2010). Association between uterine disease and indicators of neutrophil and systemic energy status in lactating Holstein cows. J. Dairy Sci. 93, 29262937. doi: 10.3168/jds.2009-2551

Gärtner, M. A., Bondzio, A., Braun, N., Jung, M., Einspanier, R., and Gabler, C. (2015). Detection and characterisation of Lactobacillus spp. in the bovine uterus and their influence on bovine endometrial epithelial cells in vitro. PLoS One 10:e0119793. doi: 10.1371/journal.pone.0119793

Gilbert, R. O., Shin, S. T., Guard, C. L., Erb, H. N., and Frajblat, M. (2005). Prevalence of endometritis and its effects on reproductive performance of dairy cows. Theriogenology 64, 1879-1888. doi: 10.1016/j.theriogenology.2005.04.022

Gurung, M., Nam, H. M., Tamang, M. D., Chae, M. H., Jang, G. C., Jung, S. C., et al. (2013). Prevalence and antimicrobial susceptibility of Acinetobacter from raw bulk tank milk in Korea. J. Dairy Sci. 96, 1997-2002. doi: 10.3168/jds.2012-5965

Heidarpour, M., Mohri, M., Fallah-Rad, A. H., Dehghan Shahreza, F., and Mohammadi, M. (2012). Acute-phase protein concentration and metabolic status affect the outcome of treatment in cows with clinical and subclinical endometritis. Vet. Rec. 171:219. doi: 10.1136/vr.100947

Jeon, S. J., Cunha, F., Vieira-Neto, A., Bicalho, R. C., Lima, S., Bicalho, M. L., et al. (2017). Blood as a route of transmission of uterine pathogens from the gut to the uterus in cows. Microbiome 5:109. doi: 10.1186/s40168-017-0328-9

Jeon, S. J., Vieira-Neto, A., Gobikrushanth, M., Daetz, R., Mingoti, R. D., Parize, A. C. B., et al. (2015). Uterine microbiota progression from calving until establishment of metritis in dairy cows. Appl. Environ. Microbiol. 81, 63246332. doi: 10.1128/AEM.01753-15

Karstrup, C. C., Agerholm, J. S., Jensen, T. K., Swaro, L. R. V., Klitgaard, K., Rasmussen, E. L., et al. (2017a). Presence and localization of bacteria in the bovine endometrium postpartum using fluorescence in situ hybridization. Theriogenology 92, 167-175. doi: 10.1016/j.theriogenology.2017.01.026

Karstrup, C. C., Klitgaard, K., Jensen, T. K., Agerholm, J. S., and Pedersen, H. G. (2017b). Presence of bacteria in the endometrium and placentomes of pregnant cows. Theriogenology 99, 41-47. doi: 10.1016/j.theriogenology.2017.05.013

Karstrup, C. C., Pedersen, H. G., Jensen, T. K., and Agerholm, J. S. (2017c). Bacterial invasion of the uterus and oviducts in bovine pyometra. Theriogenology 93, 93-98. doi: 10.1016/j.theriogenology.2017.01.027

Kasimanickam, R., Duffield, T. F., Foster, R. A., Gartley, C. J., Leslie, K. E., Walton, J. S., et al. (2004). Endometrial cytology and ultrasonography for the detection of subclinical endometritis in postpartum dairy cows. Theriogenology 62, 9-23. doi: 10.1016/j.theriogenology.2003.03.001

Knudsen, L. R. V., Karstrup, C. C., Pedersen, H. G., Agerholm, J. S., Jensen, T. K., and Klitgaard, K. (2015). Revisiting bovine pyometra-New insights into the disease using a culture-independent deep sequencing approach. Vet. Microbiol. 175, 319-324. doi: 10.1016/j.vetmic.2014.12.006

Knudsen, L. R. V., Karstrup, C. C., Pedersen, H. G., Angen, Ø, Agerholm, J. S., Rasmussen, E. L., et al. (2016). An investigation of the microbiota in uterine flush samples and endometrial biopsies from dairy cows during the first $7 \&$ 
weeks postpartum. Theriogenology 86, 642-650. doi: 10.1016/j.theriogenology. 2016.02.016

LeBlanc, S. J., Duffield, T. F., Leslie, K. E., Bateman, K. G., Keefe, G. P., Walton, J. S., et al. (2002). Defining and diagnosing postpartum clinical endometritis and its impact on reproductive performance in dairy cows. J. Dairy Sci. 85, 2223-2236. doi: 10.3168/jds.S0022-0302(02)74302-6

Liu, M., Wu, Q., Wang, M., Fu, Y., and Wang, J. (2016). Lactobacillus rhamnosus GR-1 limits Escherichia coli-induced inflammatory responses via attenuating MyD88-dependent and MyD88-independent pathway activation in bovine endometrial epithelial cells. Inflammation 39, 1483-1494. doi: 10.1007/s10753016-0382-7

Lobb, D. A., Loeman, H. J., Sparrow, D. G., and Morck, D. W. (1999). Bovine polymorphonuclear neutrophil-mediated phagocytosis and an immunoglobulin G2 protease produced by Porphyromonas levii. Can. J. Vet. Res. 63, 113-118.

Madoz, L. V., Giuliodori, M. J., Migliorisi, A. L., Jaureguiberry, M., and de la Sota, R. L. (2014). Endometrial cytology, biopsy, and bacteriology for the diagnosis of subclinical endometritis in grazing dairy cows. J. Dairy Sci. 97, 195-201. doi: $10.3168 /$ jds.2013-6836

Markusfeld, O. (1987). Periparturient traits in seven high dairy herds. incidence rates, association with parity, and interrelationships among traits. J. Dairy Sci. 70, 158-166. doi: 10.3168/jds.S0022-0302(87)79990-1

Moore, S. G., Ericsson, A. C., Poock, S. E., Melendez, P., and Lucy, M. C. (2017). Hot topic: $16 \mathrm{~S}$ rRNA gene sequencing reveals the microbiome of the virgin and pregnant bovine uterus. J. Dairy Sci. 100, 4953-4960. doi: 10.3168/jds.201712592

Nagaraja, T. G., Narayanan, S. K., Stewart, G. C., and Chengappa, M. M. (2005). Fusobacterium necrophorum infections in animals: pathogenesis and pathogenic mechanisms. Anaerobe 11, 239-246. doi: 10.1016/j.anaerobe.2005. 01.007

Pothmann, H., Prunner, I., Wagener, K., Jaureguiberry, M., de la Sota, R. L., Erber, R., et al. (2015). The prevalence of subclinical endometritis and intrauterine infections in repeat breeder cows. Theriogenology 83, 1249-1253. doi: 10.1016/j.theriogenology.2015.01.013

Prunner, I., Pothmann, H., Wagener, K., Giuliodori, M., Huber, J., EhlingSchulz, M., et al. (2014a). Dynamics of bacteriologic and cytologic changes in the uterus of postpartum dairy cows. Theriogenology 82, 1316-1322. doi: 10.1016/j.theriogenology.2014.08.018

Prunner, I., Wagener, K., Pothmann, H., Ehling-Schulz, M., and Drillich, M. (2014b). Risk factors for uterine diseases on small- and medium-sized dairy farms determined by clinical, bacteriological, and cytological examinations. Theriogenology 82, 857-865. doi: 10.1016/j.theriogenology.2014.06.015

Ribeiro, E. S., Lima, F. S., Greco, L. F., Bisinotto, R. S., Monteiro, A. P. A., Favoreto, M., et al. (2013). Prevalence of periparturient diseases and effects on fertility of seasonally calving grazing dairy cows supplemented with concentrates. J. Dairy Sci. 96, 5682-5697. doi: 10.3168/jds.2012-6335

Rotstein, O. D. (1993). Interactions between leukocytes and anaerobic bacteria in polymicrobial surgical infections. Clin. Infect. Dis. 16, S190-S194. doi: 10.1093/ clinids/16.Supplement_4.S190

Santos, T. M. A., and Bicalho, R. C. (2012). Diversity and succession of bacterial communities in the uterine fluid of postpartum metritic, endometritic and healthy dairy cows. PLoS One 7:e53048. doi: 10.1371/journal.pone.0053048

Segata, N., Izard, J., Waldron, L., Gevers, D., Miropolsky, L., Garrett, W. S., et al. (2011). Metagenomic biomarker discovery and explanation. Genome Biol. 12:R60. doi: 10.1186/gb-2011-12-6-r60
Sens, A., and Heuwieser, W. (2013). Presence of Escherichia coli, Trueperella pyogenes, $\alpha$-hemolytic streptococci, and coagulase-negative staphylococci and prevalence of subclinical endometritis. J. Dairy Sci. 96, 6347-6354. doi: 10.3168/ jds.2013-6646

Shannon, P., Markiel, A., Ozier, O., Baliga, N. S., Wang, J. T., Ramage, D., et al. (2003). Cytoscape: a software environment for integrated models of biomolecular interaction networks. Genome Res. 13, 2498-2504. doi: 10.1101/ gr.1239303

Sheldon, I. M., Cronin, J., Goetze, L., Donofrio, G., and Schuberth, H.-J. (2009). Defining postpartum uterine disease and the mechanisms of infection and immunity in the female reproductive tract in cattle1. Biol. Reprod. 81, 1025-1032. doi: 10.1095/biolreprod.109.077370

Sheldon, I. M., Lewis, G. S., LeBlanc, S., and Gilbert, R. O. (2006). Defining postpartum uterine disease in cattle. Theriogenology 65, 1516-1530. doi: 10.1016/j.theriogenology.2005.08.021

Wagener, K., Gabler, C., and Drillich, M. (2017). A review of the ongoing discussion about definition, diagnosis and pathomechanism of subclinical endometritis in dairy cows. Theriogenology 94, 21-30. doi: 10.1016/j.theriogenology.2017. 02.005

Wagener, K., Grunert, T., Prunner, I., Ehling-Schulz, M., and Drillich, M. (2014). Dynamics of uterine infections with Escherichia coli, Streptococcus uberis and Trueperella pyogenes in post-partum dairy cows and their association with clinical endometritis. Vet. J. 202, 527-532. doi: 10.1016/j.tvjl.2014. 08.023

Wagener, K., Prunner, I., Pothmann, H., Drillich, M., and Ehling-Schulz, M. (2015). Diversity and health status specific fluctuations of intrauterine microbial communities in postpartum dairy cows. Vet. Microbiol. 175, 286-293. doi: 10.1016/j.vetmic.2014.11.017

Williams, E. J., Fischer, D. P., Pfeiffer, D. U., England, G. C. W., Noakes, D. E., Dobson, H., et al. (2005). Clinical evaluation of postpartum vaginal mucus reflects uterine bacterial infection and the immune response in cattle. Theriogenology 63, 102-117. doi: 10.1016/j.theriogenology.2004.03.017

Yeoman, C. J., Ishaq, S. L., Bichi, E., Olivo, S. K., Lowe, J., and Aldridge, B. M. (2018). Biogeographical differences in the influence of maternal microbial sources on the early successional development of the bovine neonatal gastrointestinal tract. Sci. Rep. 8:3197. doi: 10.1038/s41598-018-21440-8

Zelezniak, A., Andrejev, S., Ponomarova, O., Mende, D. R., Bork, P., and Patil, K. R. (2015). Metabolic dependencies drive species co-occurrence in diverse microbial communities. Proc. Natl. Acad. Sci. U.S.A. 112, 201522642. doi: 10. 1073/pnas.1522642113

Zengler, K., and Zaramela, L. S. (2018). The Social Network of Microorganisms How Auxotrophies Shape Complex Communities. London: Nature Publishing Group.

Conflict of Interest Statement: The authors declare that the research was conducted in the absence of any commercial or financial relationships that could be construed as a potential conflict of interest.

Copyright (c) 2018 Wang, Liu, Xu, An, Wang and Zhu. This is an open-access article distributed under the terms of the Creative Commons Attribution License (CC BY). The use, distribution or reproduction in other forums is permitted, provided the original author(s) and the copyright owner(s) are credited and that the original publication in this journal is cited, in accordance with accepted academic practice. No use, distribution or reproduction is permitted which does not comply with these terms. 